\title{
AdSR Based Fault Diagnosis for Three-Axis Boring and Milling Machine
}

\author{
Bing $\mathrm{Li}^{1}{ }^{1}{ }^{*}$ - Jimeng $\mathrm{Li}^{1}$ - Jiyong Tan${ }^{2}-$ Zhengjia $\mathrm{He}^{1}$ \\ ${ }^{1}$ State Key Laboratory for Manufacturing Systems Engineering, Xi'an Jiaotong University, China \\ 2 The 29th Institute of China Electronics Technology Group Corporation, China
}

\begin{abstract}
This paper introduced an adaptive stochastic resonance (AdSR) signal processing technique to extract fault feature of machining accuracy decay in boring and milling machine providing a vibration time-frequency distribution with adaptable precision. The AdSR uses a correlation coefficient of the input signals and noise as a weight to construct the weighted kurtosis (WK) index. The influence of high frequency noise is alleviated and the index used in traditional SR is improved accordingly. The AdSR with WK can obtain optimal parameters adaptively. In addition, through the secondary utilization of noise, AdSR makes the signal output waveform smoother and the fluctuation period more obvious. It has been found that AdSR appears to be a better tool compared to fast Fourier transform for fault characterization extraction in boring and milling machine in experiment case. It has been concluded that AdSR based signal processing technology successfully diagnosis the fault of machining accuracy decay in three-axis boring and milling machine.
\end{abstract}

Keywords: stochastic resonance, fault diagnosis, boring and milling machine

\section{O INTRODUCTION}

The three-axis boring and milling machine are key devices in the modern manufacturing industry. Under operation, the components' faults degrade the machining accuracy. It is difficult to detect fault features because the structures of three-axis boring and milling machines are complex. The factors such as the influence of transmission path, the transmission medium, the ambient environment, etc., degrade the measured signals. They lower the signal-to-noise ratio. In an extreme case useful information is buried in the noise so we can hardly recover it [1]. Therefore, it is necessary to study the fault diagnosis methods of this kind of machines [2] to [5].

Recently the detection of the incipient, weak fault has attracted more and more attention. Almost all conventional methods in weak signal processing are applied to filter or mask noise [6] and [7] so that while the noise is reduced, the useful signal may be weakened or even destroyed. Different from the traditional signal processing methods, stochastic resonance (SR) as a novel signal processing method, can achieve the effect of detecting a signal by utilizing noise to amplify weak signals in nonlinear dynamical systems instead of eliminating noise. Due to the characteristics of using noise to enhance signals, SR has extensively drawn attention in wide fields, especially in the weak signals detection [8] to [10].

During the past two decades, there have been many theoretical developments of SR in bistable systems [11] to [14]. Based on adiabatic approximation theory, the classical SR is only applicable to the small parameters object, namely the driving force frequency and amplitude and noise intensity are far less than 1 [15]. But large parameter problems (driving force frequency and/or amplitude and/or noise intensity can be much larger than 1) may usually be involved in fault diagnosis of the mechanical systems. Therefore, the study of large parameter stochastic resonance methods become necessary, and in fact several achievements have been obtained during the past few years, such as modulated stochastic resonance (MSR) [16], rescaling frequency stochastic resonance (RFSR) [17], frequency-shifted and re-scaling stochastic resonance (FRSR) [18] and so on. All of these non-classical SR methods have greatly enlarged its application areas.

The occurrence of SR needs strict conditions, that is, the periodic signal, noise and the nonlinear system must satisfy certain matching relations. However, based on the research theory at present, qualitative analysis of this matching relation can be only obtained [19]. So far, the engineering application of SR, which mainly depends on researchers' experience of the and a large number of experiments has been limited to a large extent. With the development of computer technology, adaptive signal processing has been developed. Adaptive stochastic resonance (AdSR) was firstly proposed by Sanya Mitamin [20] who observed stochastic resonance with tuning the noise level. Adaptive stochastic resonance algorithms mainly contain two points: one is a study of the search rules and the other is the selection of the optimization index which is highly significant because it can determine whether the adaptive SR algorithm is valid or not. So far, among all of the measurement indexes that can evaluate the detection effect of SR, weighted kurtosis (WK) [21] and weighted signal-to-noise ratio 
(WSNR) [22] have been used most widely in signal processing and fault diagnosis.

By SR methods, the weak signals can be enhanced to a certain extent. However, when the signal-noiseratio is too low, the detection effect is not satisfactory. In order to further improve the detection effect of the weak signals, stochastic resonance enhancement methods have been studied, such as cascade stochastic resonance [23], coupled stochastic resonance [13] and so on. With the intercoupling of adjacent resonance units, coupled SR can interrelate all of resonance units to improve the output signal-noise-ratio appropriately. And in a sense, SR based on a mechanism of energy transition from high-frequency area to low-frequency area to amplify low-frequency signal gradually can be regarded as a special low-pass filter and its filtering effect is better than the conventional low-pass filter [23]. Cascade SR, two bistable systems connected in series can weaken high-frequency dithering and make the output time domain waveform more smooth. The cascade SR can achieve higher signal-noise-ratio than single SR. Therefore, in processing the weak signals, the cascade SR has more advantages.

In the present work, the classical SR theory is introduced in brief in section 1.1, and an adaptive stochastic resonance algorithm is introduced in section 1.2. Finally, AdSR is applied to fault diagnosis for boring and milling machine in section 2 . The effectiveness of the proposed method is confirmed by the application result.

\section{STOCHASTIC RESONANCE}

\subsection{Basic Theory of Stochastic Resonance}

SR, introduced by Benzi et al. [24], is a physical phenomenon. Here, for reasons of a convenient description the overdamped motion of a Brownian particle in a bistable potential in the presence of noise and periodic forcing is considered:

$$
\dot{x}(t)=-U^{\prime}(x)+A_{0} \cos (\Omega t+\varphi)+\xi(t),
$$

where $U(x)$ denotes the reflection-symmetric quadratic potential function:

$$
U(x)=-\frac{a}{2} x^{2}+\frac{b}{4} x^{4},
$$

where the barrier parameters $a$ and $b$ are positive real parameters.

Then Eq. (1) can be written as:

$$
\dot{x}(t)=a x-b x^{3}+A_{0} \cos (\Omega t+\varphi)+\xi(t) .
$$

In Eq. (3), $A_{0}$ is the periodic input signal amplitude, $\Omega\left(=2 \pi f_{0}\right)$ is the driving frequency, $\xi(t)$ denotes a zero-mean, Gaussian white noise, i.e.,

$$
\begin{aligned}
\langle\xi(t)\rangle & =0, \\
\langle\xi(t) \xi(t+\tau)\rangle & =2 D \delta(\tau),
\end{aligned}
$$

here, $D$ is the noise intensity, $\langle *\rangle$ stands for the statistical mean value calculation. According to the Eq.(2), there are two stable fixed points at $x= \pm \sqrt{a / b}$ and one quasi-stable fixed point at $x=0$ which are the local minima and local maximum of the potential function $U(x)$ respectively. The height of the potential barrier of the two minima is $\Delta U=a^{2} / 4 b$. The potential function $U(x)$ is shown in Fig. 1.

In the absence of the periodic signal and noise, the position of the Brownian particle is determined by the initial conditions and is never changed. Only in the presence of the periodic signal, the Brownian particle moves in one of the two potential wells and can not cross the barrier, however, in the assistance of noise, the particle will accumulate enough energy to cross the potential barrier. When the noise intensity is large enough, the particle will continue to cross the barrier and jump between the two potential wells back and forth. When the transition rate between the two potential wells caused by noise, namely Kramers rate $\boldsymbol{r}_{K}$, matches the period of the input signal, the periodic signal is enhanced and stochastic resonance takes place.

$$
r_{K}=\frac{a}{\sqrt{2} \pi} \exp \left(-\frac{\Delta U}{D}\right),
$$

and the requirements of the time matching in stochastic resonance:

$$
\frac{2}{r_{K}}=\frac{1}{f_{0}} .
$$

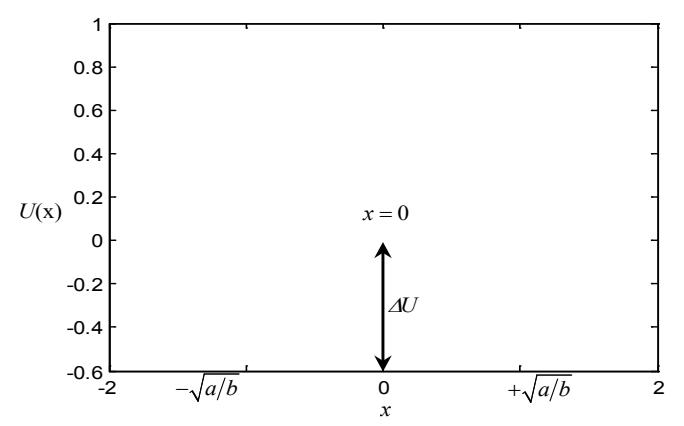

Fig. 1. The curve of the quadratic bistable potential function $U(x)$

When the amplitude of the periodic signal is smaller than the noise intensity, detection effect of the 
input signal using single stochastic resonance is not satisfactory, and the output signal still contains a certain amount of noise and the feature of the useful signal is not significant. To further improve the detection effect of the weak signal, stochastic resonance enhancement methods have been studied. Cascade stochastic resonance, single bistable system connected in series, which not only makes the amplitude of characteristic frequency more outstanding in the frequency domain, but also the output waveform becomes more smooth in the time domain. The framework of cascade stochastic resonance, two bistable systems connected in series, is shown in Fig. 2.

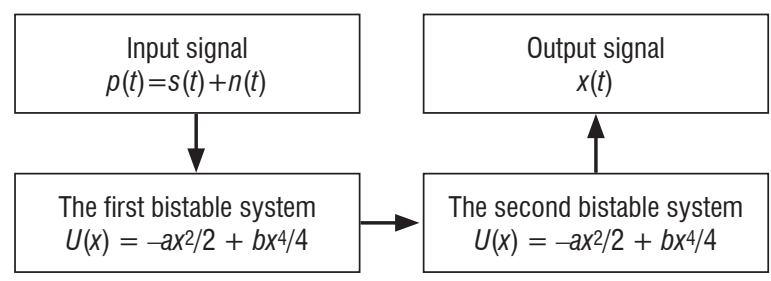

Fig. 2. The framework of cascade stochastic resonance system

\subsection{Adaptive Stochastic Resonance}

Generally, an adaptive algorithm contains two aspects of contents: one is the study of search rules and the other is the selection of optimization index, which is highly significant as it can determine whether or not the adaptive algorithm is valid. In terms of SR, in all measurement indexes that can evaluate the detection effect of stochastic resonance, the signal-noise-ratio has been used most widely. The algorithm [21] flow of the adaptive stochastic resonance is shown in Fig. 3.

\section{Algorithm:}

(i) The initial values of the barrier parameters, $a, b$, theirs searching range and step size are set.

(ii) The original input signal is input into the bistable system and the output can be calculated.

(iii) Aiming at the barrier parameters in each group, $W K$ of the output signal will be calculated.

(iv) The maximum of the $W K$ will be searched in the searching range of $a$ and $b$. When $a$ and $b$ exceed the searching range, the maximum of the $W K$ and its corresponding barrier parameters $a$ and $b$ will be saved. However, if $a$ and $b$ do not exceed the searching range, the values of $a$ and $b$ will be changed and the algorithm will return to step (iii).

(v) The optimal detection result of the original input signal can be achieved by the cascade stochastic resonance by using the optimal parameters.

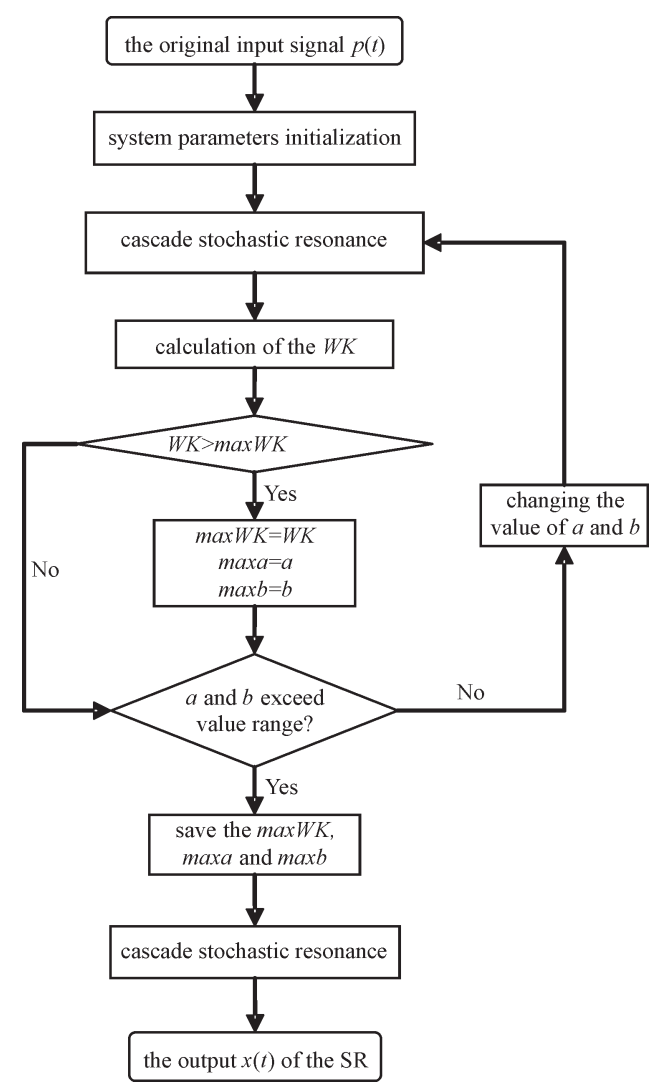

Fig. 3. The algorithm flow of the adaptive stochastic resonance

\section{FAULT DIAGNOSIS OF BORING AND MILLING MACHINE USING AdSR}

Fig. 4a is a picture of a three-axis boring and milling machine. The device was first used in 1993 when it was used to machine parts with high precision. Now, its machining precision decreases quickly. It can only serve as semi-precision machining equipment. Fig. 4b shows a bomb case of a tank which is processed by this machine.

The structural sketch of a bomb case is shown in Fig. 5. The main machining procedures include:

(i) milling end face A;

(ii) boring hole 1 ;

(iii) rotating the bomb case with $180^{\circ}$, and milling end face B;

(iv) boring hole 2 .

We found that coaxiality of two holes during a set of workpieces was poor. The capacity factor of procedure, $C p$ is 0.95 , which belongs to third lever. After adjustment of machining parameters, the coaxiality still can not approach accuracy requirement. The milled bottom face was used as allocation 
character of boring hole, so there is an error transfer effect of two procedures. The experimental results show that the location error of the milling procedure is key factor that causes coaxiality error along $x$ direction of the two holes in the boring procedure. The procedures were adjusted to another boring machine, the errors were in acceptable domain, and the capacity factor of procedure, $C p$ is approach to second lever. The results indicate that the reason for error of the three-axis boring and milling machine is self-accuracy decay. We doubt that the key factor is turning accuracy decay of machine operating platform.

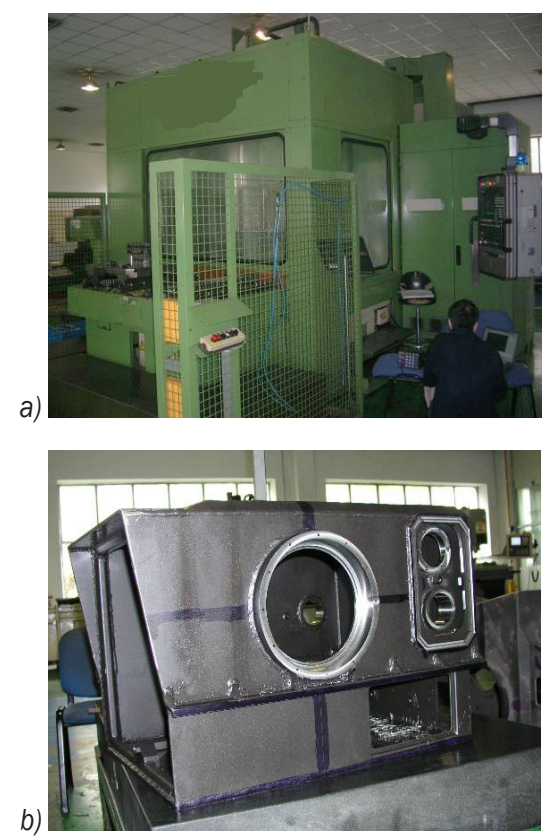

Fig. 4. Machine and it's part; a) boring and milling machine, b) a bomb case of tank

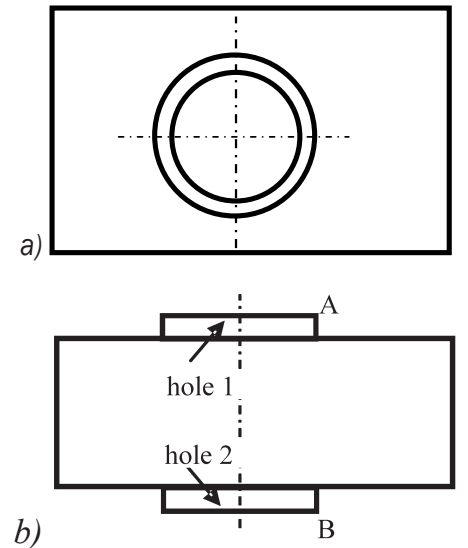

Fig. 5. Structural figure of bomb case; a) front view, b) top view

The turning operation platform is shown in Fig. 6. The platform includes mechanisms for secondary change-speed. The transmission ration of the belt is 2.5. The number of teeth on worm, $z 1$ is 1 , and the number of teeth on worm wheel, $z 2$ is 72 . Worm wheel shaft is an output one to drive the turning operation platform. Thus, the accuracy of turning operation platform depends on the accuracy of the worm couple.

The vibration signals include abundant information and are easy to be picked, so these kinds of signals are used widely in advanced measurements. In this paper, we chose the vibration data as the target detection signals. The piezoelectric accelerometer with three directions was installed on the platform. The location of sensor is shown in Fig. 7. The concrete parameters of the applied acceleration sensor are shown in Table 1.

Table 1. Detailed information of the used sensor

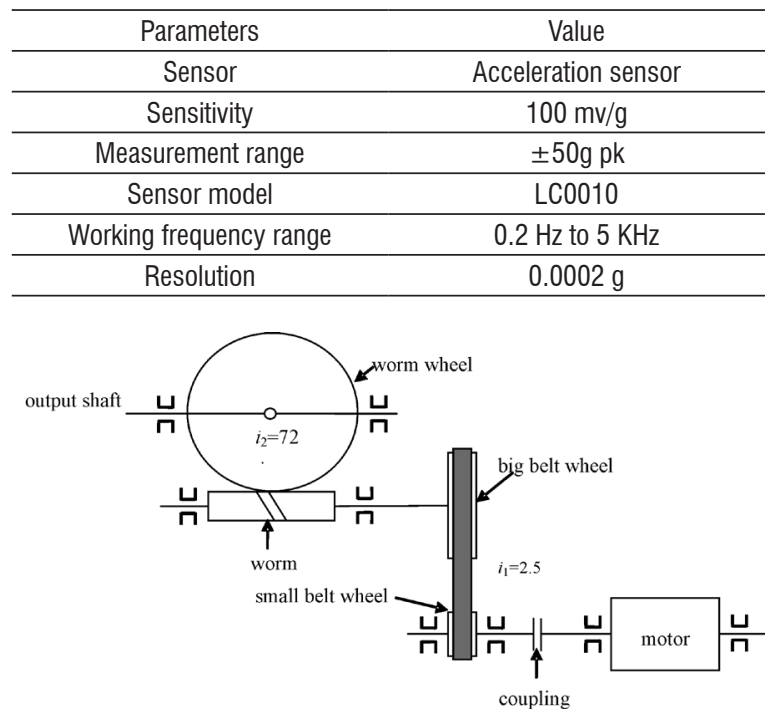

Fig. 6. The turing operationg platform

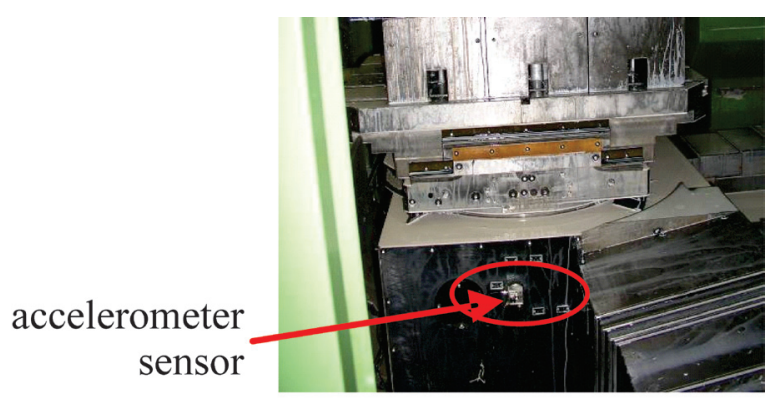

Fig. 7. Location of sensor

Sony Ex data acquisition system were utilized in this test. The speed of turning operation platform is $2000 \mathrm{rpm}$. The rotating frequency of the small belt wheel is $33.33 \mathrm{~Hz}$, and the big belt wheel and worm $13.33 \mathrm{~Hz}$. The rotating frequency of the worm wheel, 
output shaft are $0.19 \mathrm{~Hz}$. The sample frequency is set $12.8 \mathrm{kHz}$, and the length of the data is 16384 in signal processing. Fig. 8 is $x$ direction vibration signal of turning operation platform. It can be seen that the signal of time domain is in disorder. Almost no useful information can be found. The spectrum peaks of $857.80 \mathrm{~Hz}, 1523.00 \mathrm{~Hz}$ in frequency domain waveform have no relation with characteristic frequencies of transmission mechanism. The detail signal under $100 \mathrm{~Hz}$ is zoomed in and likewise there is no useful information. In Fig. 9, it can be seen that the frequency components are very complicated and still concentrate on the medium and high frequency band, which is not consistent with the fault characteristic information to be identified. Although the low frequency components can also be found in the low frequency band, the fault characteristics frequency is not obvious enough and is still polluted by the interference noise. Consequently, it is difficult to draw a final conclusion.
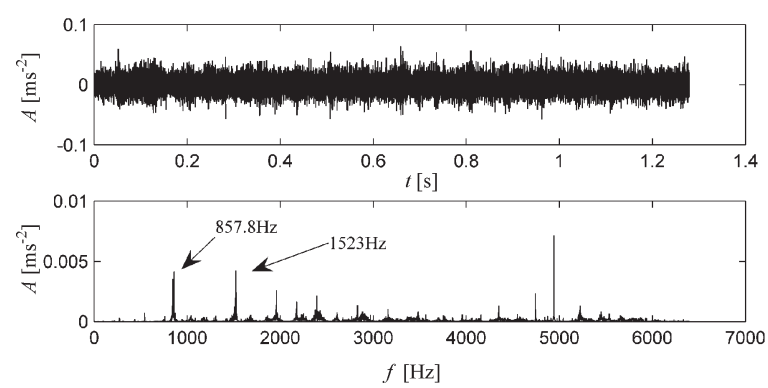

Fig. 8. Signal of platform in time and frequency domain

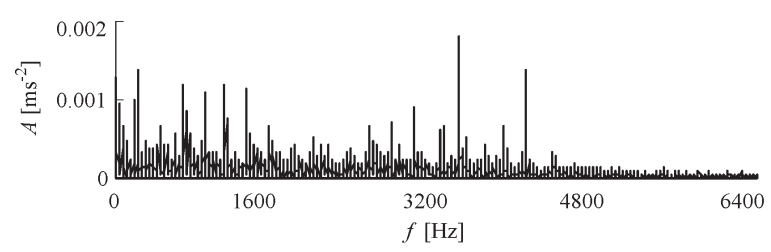

Fig. 9. Hilbert envelope spectrum of original signal

The AdSR are used to analyze the signal. The parameters of AdSR are set as below: $a$ is [0.1,5], step is $0.01, b$ is 0.1 , and the compressibility of variable metric $R$ is 200 .

By AdSR, the optimum parameter $a=4.14$ can be obtained. The optimum result of resonance domain is shown in Fig. 10. From Fig. 10, it can be seen that amplitude modulation is obvious. There are 17 waveforms of amplitude modulation with obvious periodic property. In order to get a better detection effect, we construct dipole cascade SR is constructed. Fig. 11 is the result of dipole cascade SR. After comparison of Fig. 10 and Fig. 11, it can be seen that the phenomenon of amplitude modulation in Fig. 10 is more obvious.

In order to diagnose the fault of turning operation platform, the signal in Fig. 7 is dealt with Hilbert envelope demodulation. Fig. 12 is the result of envelope demodulation. It can be seen that the periodic property is clear in Fig. 12 and corresponding frequency is $13.49 \mathrm{~Hz}$.

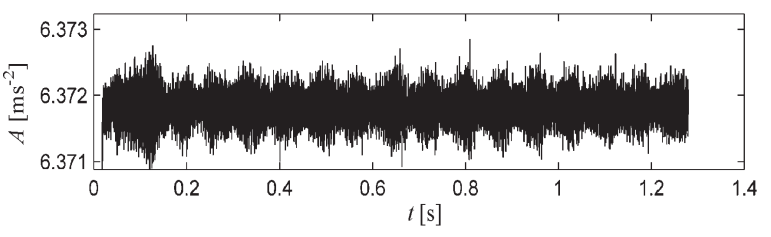

Fig. 10. Output signal of $S R$

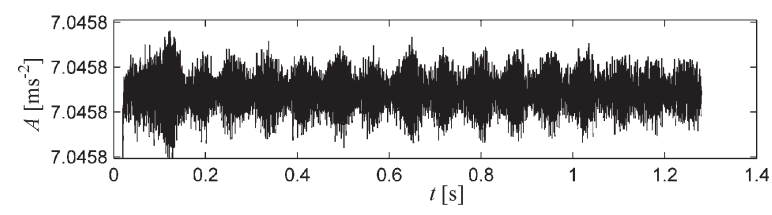

Fig. 11. Output signal of dipole cascade SR
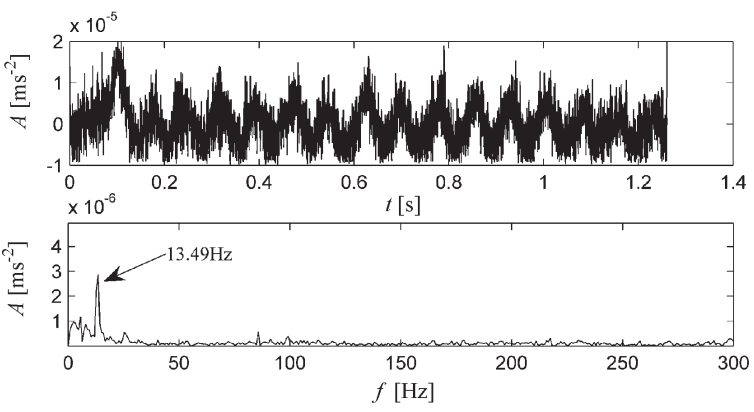

Fig. 12. Hilbert envelope demodulation of dipole cascade

$S R$

The turning operation platform is mechanism for secondary change-speed. The connecting form of the motor and worm is a flexible belt connector driving, so the fault of the motor can not influence the turning accuracy of output shaft. In addition, there is no operating frequency of motor in frequency spectrum and envelope spectrum. While the periodic envelope signal is obvious in AdSR and dipole cascade SR. We have concluded that the reason for machining error came from two aspects. The actual operation frequency of the motor is not a stable value, $2000 \mathrm{rpm}$, but that it fluctuates. On the other hand, the error is caused by frequency distinguish ability. Due to the fact that the connector of the big belt wheel and belt is flexible contact, the fault of the wheel and gear will not generate catastrophe burst. The $13.49 \mathrm{~Hz}$ frequency peak can be seen in Fig. 12 as the worm's 
characteristic frequency. The worm wear fault of the turning operation platform is concluded to be the main reason generating accuracy decay of the three-axis boring and milling machine.

\section{CONCLUSIONS}

This paper attempted to findfault features of machining accuracy decay for a boring and milling machine using the adaptive stochastic resonance method. The AdSR is found to be a better tool for extracting the fault features compaed with the Fourier analysis alleviating the influence of high frequency noise consisting primarily in the machining vibration signals. Through the secondary utilization of noise, AdSR makes the output waveform smoother and the fluctuation period more obvious, the signal-noise-ratio is further improved, and realizes the enhancement of the fault feature. Research is being continued to explore the changing regularities of the machine fault diagnosis using the AdSR to monitor continuous machining procedure of boring and milling machine.

\section{ACKNOWLEDGEMENTS}

This work was supported by the National Basic Research Program of China ("973" Program) (Grant No. 2011CB706805) and the National Natural Science Foundation of China (Grant No. 11176024, 51075033).

\section{REFERENCES}

[1] Hu, N.Q., Chen, M., Wen, X.S. (2003). The application of stochastic resonance theory for early detecting rubimpact fault of rotor system. Mechanical Systems and Signal Processing, vol. 17, no. 4, p. 883-895, DOI:10.1006/mssp.2002.1470.

[2] Čuš, F., Župerl, U. (2011). Real-Time cutting tool conditon monitoring in milling. Strojniški vestnik Journal of Mechanical Engineering, vol. 57, no. 2, p. 142-150, DOI:10.5545/sv-jme.2010.079.

[3] Tuan, D.V., Pil, C.U. (2011). Signal model based fault detection and diagnosis for induction motors using features of vibration signal in two dimension domain. Strojniski vestnik - Journal of Mechanical Engineering, vol. 57, no. 9, p. 655-666.

[4] Chaari, R., Abdennadher, M., Louati, J., Haddar, M. (2011). Modelling of the 3D Machining Geometric Defects Accounting for Workpiece Vibratory Behaviour. International Journal of Simulation Modelling, vol. 10, no. 2, p. 66-77, DOI:10.2507/IJSIMM10(2)2.173.

[5] Roy, S.S. (2010). Modelling of Tool Life, Torque and Thrust Force in Drilling: a Neuro-Fuzzy Approach. International Journal of Simulation Modelling, vol. 9, no. 2, p. 74-85, DOI:10.2507/IJSIMM09(2)2.149.
[6] Jiang, H.K., He, Z.J., Duan C.D. (2006). Gearbox fault diagnosis using adaptive redundant lifting scheme. Mechanical Systems and Signal Processing, vol. 20, no. 8, p. 1992-2006, DOI:10.1016/j.ymssp.2005.06.001.

[7] Li, B., Chen, X.F., He, Z.J. (2005). Detection of crack location and size in structures using wavelet finite element methods. Journal of Sound and Vibration, vol. 285, no. 4-5, p. 767-782, DOI:10.1016/j. jsv.2004.08.040.

[8] Hou, Z.F., Yang, J., Wang, Y.P. (2008). Weak signal detection based on stochastic resonance combining with genetic algorithm. 11th IEEE Singapore International Conference on Communication Systems, IEEE ICCS, p. 484-488.

[9] Zhao, W.L., Wang, Z.G., Huang, Z.Q. (2011). A new model of stochastic resonance used in weak signal detection. Applied Mechanics and Materials, vol. 43, p. 229-232, DOI:10.4028/www.scientific.net/ AMM.43.229.

[10] Chen, M., Hu, N.Q., Qin, G.J. (2008). A study on additional-signal-enhanced stochastic resonance in detecting weak signals. IEEE International Conference on Networking, Sensing and Control, IEEE ICNSC, p. 1636-1640.

[11] Ye, Q.H., Huang, H.N., He, X.Y. (2003). A study on the parameters of bistable stochastic resonance systems and adaptive stochastic resonance. Proceeding of the IEEE International Conference on Robotics, Intelligent Systems and Signal, p. 484-488.

[12] Guo, W.N., Obermayer, K. (2003). Activity driven adaptive stochastic resonance. Physical Review Letters, vol. 90, p. 120602/1-120602/4.

[13] Kenfack, A., Kamal, P.S. (2010). Stochastic resonance in coupled underdamped bistable systems. Physical Review E - Statistical, Nonlinear, and Soft Matter Physics, vol. 82, no. 4, p. 046224/1-046624/5.

[14] Zhou, Y.R. (2011). Stochastic resonance in a time-delayed mono-stable system with correlated multiplicative and additive white noise. Chinese Physics B, vol. 1, no. 20, p. 010501/1-010501/6.

[15] Leng, Y.G., Wang, T.Y., Qin, X.D. (2004). Power spectrum research of twice sampling stochastic resonance in a bistable system. Acta physical Sinica, vol. 53, p. 717-723.

[16] Ye, Q.H., Huang, H.N., He, X.Y. (2003). Improved bearing estimates of weak signals using stochastic resonance and frequency shift techniques. MTS/IEEE Oceans Conference Record, vol. 5, no. 3, p. 2410-2413.

[17] Leng, Y.G., Wang, T.Y., Guo, Y. (2007). Engineering signal processing based on bistble stochastic resonance. Mechanical Systems and Signal Processing, vol. 21, no. 1, p. 138-150, DOI:10.1016/j.ymssp.2005.08.002.

[18] Tan, J.Y., Chen, X.F., Wang, J.Y. (2009). Study of frequency-shifted and re-scaling stochastic resonance and its application to fault diagnosis. Mechanical systems and signal processing, vol. 23, no. 3, p. 811822, DOI:10.1016/j.ymssp.2008.07.011. 
[19] Xu, B.H., Duan, F.B., Bao, R.H. (2002). Stochastic resonance with tuning system parameters: the application of bistable system in signal processing. Chaos Solitons Fractals, vol. 13, no. 4, p. 633-644, DOI:10.1016/S0960-0779(00)00266-6.

[20] Mitamin, S., Kosko, B. (1998). Adaptive stochastic resonance. Proceedings of IEEE, vol. 86, no. 11, p. 2152-2183, DOI:10.1109/5.726785.

[21] Tan, J.Y., Chen, X.F., He, Z.J. (2010). Impact signal detection method with adaptive stochastic resonance. Journal of Mechanical Engineering, vol. 46, no. 23, p. 61-67, DOI:10.3901/JME.2010.23.061. (In Chinese)
[22] Li, B., Li, J.M., He, Z.J. (2011). Fault feature enhancement of gearbox in combined machining center by using adaptive cascade stochastic resonance. Science China Technological Sciences, vol. 54, no. 12, p. 3203-3210, DOI:10.1007/s11431-011-4612-9.

[23] He, H.L., Wang, T.Y., Leng, Y.G. (2007). Study on nonlinear filter characteristic and engineering application of cascaded bistable stochastic resonance system. Mechanical systems and signal processing, vol. 7, no, 21, p. 2740-2749, DOI:10.1016/j.ymssp.2007.02.004.

[24] Benzi, R., Sutera, A., Vulpiana, A. (1981). The mechanism of stochastic resonance. Physical A, vol. 14, no. 11, p. 453-457. 